\title{
Antimicrobial susceptibility/resistance and NG-MAST characterisation of Neisseria gonorrhoeae in Belarus, Eastern Europe, 2010-2013
}

Fiodar Lebedzeu', Daniel Golparian², Leonid Titov', Nataliya Pankratava³, Slavyana Glazkova', Irina Shimanskaya ${ }^{4}$, Natallia Charniakova ${ }^{5}$, Aliaksandr Lukyanau ${ }^{4}$, Marius Domeika ${ }^{6}$ and Magnus Unemo ${ }^{2^{*}}$

\begin{abstract}
Background: Gonorrhoea and widely spread antimicrobial resistance (AMR) in its etiological agent Neisseria gonorrhoeae are major public health concerns worldwide. Gonococcal AMR surveillance nationally and internationally, to identify emerging resistance and inform treatment guidelines, is imperative for public health purposes. In 2009, AMR surveillance was initiated in Belarus, Eastern Europe because no gonococcal AMR data had been available for at least two decades. Herein, the prevalence and trends of gonococcal AMR and molecular epidemiological characteristics of N. gonorrhoeae strains from 2010 to 2013 in Belarus, are described.

Methods: N. gonorrhoeae isolates ( $n=193)$ obtained in the Mogilev $(n=142)$, Minsk $(n=36)$ and Vitebsk $(n=15)$ regions of Belarus in 2010 ( $n=72), 2011$ (n=6), 2012 ( $n=75)$ and $2013(n=40)$ were analyzed in regards to AMR using the Etest method and for molecular epidemiology with N. gonorrhoeae multi-antigen sequence typing (NG-MAST).

Results: During 2010-2013, the proportions of resistant N. gonorrhoeae isolates were as follows: tetracycline 36\%, ciprofloxacin $28 \%$, penicillin G 9\%, azithromycin $5 \%$, and cefixime $0.5 \%$. Only one (0.5\%) $\beta$-lactamase producing isolate was detected. No isolates resistant to ceftriaxone and spectinomycin were identified. Overall, the resistance levels to tetracycline, ciprofloxacin and penicillin $\mathrm{G}$ were relatively stable. Interestingly, the level of resistance to azithromycin declined from 12\% in 2010 to 0\% in 2013 (P<0.05). In total, 70 NG-MAST STs were identified. The predominant STs were ST1993 ( $n=53)$, ST807 $(n=13)$, ST285 $(n=8)$ and ST9735 $(n=8)$. Many novel STs $(n=43,61 \%)$, representing $41 \%$ of all isolates, were found.

Conclusions: During 2010-2013, the N. gonorrhoeae population in Belarus displayed high and relatively stable resistance levels to tetracycline, ciprofloxacin, and penicillin $G$, while the resistance to azithromycin declined. One isolate was resistant to cefixime, but no resistance to ceftriaxone or spectinomycin was found. The results of the present surveillance initiated in 2009 were also used to replace penicillin G with ceftriaxone $(1 \mathrm{~g}$ single dose intramuscularly) as the first-line drug for empiric treatment of gonorrhoea in the national treatment guidelines in Belarus in late 2009. It is essential to further strengthen the surveillance of gonococcal AMR and ideally survey also treatment failures and molecular epidemiological genotypes in Belarus.
\end{abstract}

Keywords: Neisseria gonorrhoeae, Gonorrhoea, Antimicrobial resistance, Surveillance, Extended-spectrum cephalosporins (ESCs), Ceftriaxone, Cefixime, Treatment, N. gonorrhoeae multiantigen sequence typing (NG-MAST), Belarus

\footnotetext{
* Correspondence: magnus.unemo@orebroll.se

${ }^{2}$ WHO Collaborating Centre for Gonorrhoea and other Sexually Transmitted

Infections, Swedish Reference Laboratory for Pathogenic Neisseria,

Department of Laboratory Medicine, Microbiology, Örebro University

Hospital, Örebro, Sweden

Full list of author information is available at the end of the article
}

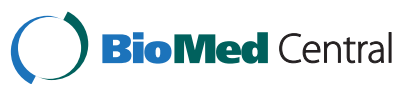

(c) 2015 Lebedzeu et al.; licensee BioMed Central. This is an Open Access article distributed under the terms of the Creative Commons Attribution License (http://creativecommons.org/licenses/by/4.0), which permits unrestricted use, distribution, and reproduction in any medium, provided the original work is properly credited. The Creative Commons Public Domain Dedication waiver (http://creativecommons.org/publicdomain/zero/1.0/) applies to the data made available in this article, unless otherwise stated. 


\section{Background}

Gonorrhoea, etiological agent Neisseria gonorrhoeae, is a public health concern globally. In most of the nonEuropean Union (EU)/European Economic Area (EEA) countries $(n=23)$, which mostly represent independent countries from the former Soviet Union and Yugoslavic Republic, of the World Health Organization (WHO) European Region (53 countries), the gonorrhoea incidence has rapidly declined during the last 20 years. In the Eastern European non-EU/EEA country Belarus (9.5 million inhabitants), the incidence has also mainly declined during the last two decades. However, the incidence remains high and in 2011 Belarus reported the third highest incidence (35.4 per 100000 population) in the WHO European Region ([1,2],http://data.euro.who.int/cisid). The reported gonorrhoea incidences in Belarus might also be underestimated, which is due to suboptimal diagnostics, access to testing, case reporting, e.g. lack of reporting of cases diagnosed in the private sector, and surveillance [2-4].

$N$. gonorrhoeae has developed antimicrobial resistance (AMR) to all drugs previously recommended for treatment of gonorrhoea. In vitro (low-level and high-level) and rare treatment failures have now emerged to the last remaining option for first-line empirical monotherapy in most countries globally, that is, the extended-spectrum cephalosporin (ESC) ceftriaxone [5-18]. Therefore, enhanced and quality assured surveillance of gonococcal AMR, nationally and internationally, is essential to monitor the AMR trends, identify emerging AMR and inform a regular update of the STI management and treatment guidelines locally, nationally and globally. This is in strict concordance with the global action plan and European response plan to combat gonococcal AMR published by the WHO $[19,20]$ and the European Centre for Disease Prevention and Control (ECDC) [21], respectively.

Worryingly, in the non-EU/EEA countries of the WHO European Region, quality assured gonococcal AMR surveillance only exist in $13 \%(3 / 23)$ of the countries [2]. In 2009, because no gonococcal AMR data had been available for at least two decades gonococcal AMR surveillance was initiated in Belarus by the Eastern European Network for Sexual and Reproductive Health [22] in collaboration with the WHO. This AMR surveillance has been quality assured in accordance with WHO standards and the 2008 WHO N. gonorrhoeae reference strains are used as quality controls [23].

The aims of the present study were to describe the prevalence and trends of gonococcal AMR and molecular epidemiological characteristics of $N$. gonorrhoeae strains from 2010 to 2013 in Belarus.

\section{Methods}

\section{Study population}

$N$. gonorrhoeae isolates $(\mathrm{n}=193)$ were obtained at the Mogilev Regional ( $n=142)$, Minsk City $(n=36)$ and
Vitebsk Regional ( $\mathrm{n}=15)$ Dermato-Venerological Dispensaries in Belarus in $2010(n=72), 2011(n=6), 2012$ $(n=75)$ and $2013(n=40)$. Mainly consecutive culture positive gonorrhoea patients from January 2010 to April 2013 were included. Urethral and cervical specimens from females and urethral specimens from males were collected.

All specimens were cultured on selective gonococcal agar media and suspected gonococcal colonies were subsequently confirmed as $N$. gonorrhoeae by colony morphology, Gram staining, oxidase test and a carbohydrate utilization test [24]. The isolates were stored in cryogenic storage dewar with liquid nitrogen $\left(-196^{\circ} \mathrm{C}\right)$ or in ultra-low temperature freezer $\left(-70^{\circ} \mathrm{C}\right)$ in preservation media and then shipped on dry ice to the WHO Collaborating Centre for Gonorrhoea and other STIs, Sweden for further analysis. All examined gonococcal isolates were cultured and stored as part of the routine diagnostics (standard care) and no patient identification information was available in the study.

\section{Antimicrobial susceptibility and $\beta$-lactamase testing}

The minimum inhibitory concentration (MIC; $\mathrm{mg} / \mathrm{L}$ ) of ceftriaxone, cefixime, azithromycin, spectinomycin, ciprofloxacin, penicillin $\mathrm{G}$, tetracycline and gentamicin was analysed using the Etest methodology on Difco GC Medium Base (Becton, Dickinson and Company, Sparks, MD, USA) supplemented with $1 \%$ BBL IsoVitaleX Enrichment (Becton, Dickinson and Company, Sparks, MD, USA), with agar plates incubated for 18-20 h at $35-37^{\circ} \mathrm{C}$ in a $5 \% \mathrm{CO}_{2}$-enriched atmosphere, according to the instructions from the manufacturer (bioMérieux $A B$, Solna, Sweden). The Etest results were interpreted as susceptible (S), intermediate susceptible (I) and resistant (R) according to the breakpoints stated by The European Committee on Antimicrobial Susceptibility Testing (EUCAST; www.eucast.org/clinical_breakpoints). For gentamicin, no breakpoints are stated by any organization and, accordingly, only $\mathrm{MIC}$ range, $\mathrm{MIC}_{50}$ and $\mathrm{MIC}_{90}$ were used.

$\beta$-lactamase production was identified with nitrocefin solution $(0.5 \mathrm{~g} / \mathrm{mL})$, according to the manufacturer's instructions (Oxoid, Basingstoke, Hants, England).

\section{Isolation of genomic DNA}

Genomic DNA was isolated using the NorDiag Bullet robot (NorDiag ASA Company, Oslo, Norway) with BUGS'n BEADS STI-fast kit (NorDiag ASA Company, Oslo, Norway), according to the manufacturer's instructions.

\section{Molecular epidemiological typing}

NG-MAST $[25,26]$ was performed as previously described [27]. NG-MAST allele numbers of the more variable segments of por $B$ and $t b p B$, and sequence types (STs) were 
assigned using the NG-MAST website (www.ng-mast. net).

\section{Statistical analysis}

Statistical analysis was performed using the Statistica software version 9.0. Z-test for comparison of proportions was used. The level of significance was set at $P<0.05$.

\section{Results}

\section{Patient characteristics}

N. gonorrhoeae isolates (one isolate per patient) from 193 patients; 169 (87.6\%) males and 24 (12.4\%) females, were examined. The mean age for the males was 26.9 years (median age: 25.0 years; range: 19 to 57 years) and for the females 27.3 years (median age: 28.5 years; range: 19 to 33 years). The male/female ratio and age distribution was relatively similar during the four years investigated.

\section{Antimicrobial susceptibility of $N$. gonorrhoeae isolates in 2010-2013 $(n=193)$ in Belarus}

The overall antimicrobial susceptibility of all isolates is summarized in Table 1.

Briefly, the levels of in vitro resistance during 20102013 were as follows: tetracycline $36 \%$, ciprofloxacin $28 \%$, penicillin G 9\%, azithromycin $5 \%$, and cefixime $0.5 \%$ (one isolates with an MIC of $0.25 \mathrm{mg} / \mathrm{L})$. Only one $(0.5 \%)$ $\beta$-lactamase producing isolate was found. No isolates resistant to ceftriaxone and spectinomycin were identified. Overall, the resistance levels to tetracycline, ciprofloxacin and penicillin G were relatively stable. Interestingly, the level of resistance to azithromycin significantly declined from $12 \%$ in 2010 to $0 \%$ in $2013(\mathrm{P}<0.05)$. For gentamicin (MIC range: 0.25$16 \mathrm{mg} / \mathrm{L}$ ), the MICs were relatively low and all isolates considered susceptible (Table 1).

The proportion of isolates with ceftriaxone $\mathrm{MIC}<$ $0.002 \mathrm{mg} / \mathrm{L}$ was $44 \%, 67 \%$, and $63 \%$ in 2010/2011, 2012 and 2013, respectively, and in general the annual MIC distribution for ceftriaxone appeared to shift to lower MICs during the study period 2010-2013 (Figure 1). However, although no resistance to ceftriaxone was identified, in total $3.1 \%$ (5\% in 2013) had a ceftriaxone $\mathrm{MIC}$ of $0.125 \mathrm{mg} / \mathrm{L}$, which is exactly at the resistance breakpoint stated by the EUCAST (www.eucast.org/ clinical_breakpoints). Gonococcal isolates with these MICs have previously resulted in ceftriaxone treatment failures and can be considered to have a decreased susceptibility [12-14,16-18].

Table 1 Antimicrobial susceptibility of Neisseria gonorrhoeae isolates $(n=193)$ from Belarus, 2010-2013

\begin{tabular}{|c|c|c|c|c|c|c|c|c|c|c|}
\hline \multirow[t]{2}{*}{ Antimicrobial } & \multirow{2}{*}{$\begin{array}{l}\mathrm{MIC}_{50} / \\
\mathrm{MIC}_{90} / \\
\text { MIC range } \\
\text { (mg/L) }\end{array}$} & \multicolumn{3}{|c|}{ Susceptible (\%) } & \multicolumn{3}{|c|}{ Intermediate susceptible (\%) } & \multicolumn{3}{|c|}{ Resistant (\%) } \\
\hline & & $\begin{array}{l}2010-2011 \\
(n=78)^{a}\end{array}$ & $\begin{array}{l}2012 \\
(n=75)\end{array}$ & $\begin{array}{l}2013 \\
(n=40)\end{array}$ & $\begin{array}{l}2010-2011 \\
(n=78)^{a}\end{array}$ & $\begin{array}{l}2012 \\
(n=75)\end{array}$ & $\begin{array}{l}2013 \\
(n=40)\end{array}$ & $\begin{array}{l}2010-2011 \\
(\mathrm{n}=78)^{a}\end{array}$ & $\begin{array}{l}2012 \\
(n=75)\end{array}$ & $\begin{array}{l}2013 \\
(n=40)\end{array}$ \\
\hline \multirow[t]{2}{*}{ Penicillin $G^{b}$} & $0.032 / 1 /$ & - & 88 & 78 & - & 5 & 10 & - & 7 & 13 \\
\hline & $0.008-2$ & & & & & & & & & \\
\hline \multirow[t]{2}{*}{ Ceftriaxone $^{b}$} & $0.008 / 0.047 /$ & 100 & 100 & 100 & NA & NA & NA & 0 & 0 & 0 \\
\hline & $<0.002-0.125$ & & & & & & & & & \\
\hline \multirow[t]{2}{*}{ Cefixime $^{b}$} & $0.023 / 0.047 /$ & 100 & 100 & 97.5 & NA & NA & NA & 0 & 0 & 2.5 \\
\hline & $<0.016-0.25$ & & & & & & & & & \\
\hline \multirow[t]{2}{*}{ Azithromycin ${ }^{b}$} & $0.19 / 0.38 /$ & 65 & 83.3 & 90 & 23 & 15.3 & 10 & 12 & 1.3 & 0 \\
\hline & $0.032-1$ & & & & & & & & & \\
\hline \multirow[t]{2}{*}{ Ciprofloxacin ${ }^{b}$} & $0.006 / 6 /$ & 64 & 79 & 72 & 1 & 0 & 0 & 35 & 21 & 28 \\
\hline & $0.003->32$ & & & & & & & & & \\
\hline \multirow[t]{2}{*}{ Tetracycline $^{b}$} & $0.38 / 24 /$ & 56 & 56 & 55 & 8 & 9 & 5 & 36 & 35 & 40 \\
\hline & $0.094-24$ & & & & & & & & & \\
\hline Spectinomycin $^{b}$ & 12/16/3-16 & 100 & 100 & 100 & NA & NA & NA & 0 & 0 & 0 \\
\hline \multirow[t]{2}{*}{ Gentamicin $^{c}$} & 2010-2011: & & & & 2012: & & & 2013: & & \\
\hline & \multicolumn{4}{|c|}{$\begin{array}{l}\text { MIC range: } 0.25-16 \text { mg/L; } M I C_{50:} 2 \text { mg/L; } M C_{90:} \\
4 \mathrm{mg} / \mathrm{L}\end{array}$} & \multicolumn{3}{|c|}{$\begin{array}{l}\text { MIC range: } 2-16 \mathrm{mg} / \mathrm{L} ; \mathrm{MIC}_{50}: 4 \mathrm{mg} / \mathrm{L}_{i} \\
\mathrm{MIC}_{90}: 8 \mathrm{mg} / \mathrm{L}\end{array}$} & \multicolumn{3}{|c|}{$\begin{array}{l}\text { MIC range: } 3-12 \mathrm{mg} / \mathrm{L}_{;} \mathrm{MIC}_{50}: 6 \mathrm{mg} / \mathrm{L} \\
\mathrm{MIC}_{90:} 8 \mathrm{mg} / \mathrm{L}\end{array}$} \\
\hline
\end{tabular}

$\mathrm{MIC}_{50}$, minimum inhibitory concentration of $50 \%$ of isolates; $\mathrm{MIC}_{90}$, minimum inhibitory concentration of $90 \%$ of isolates; - , Not tested; NA, not applicable. ${ }^{a}$ Due to the few isolates obtained in 2011, isolates from $2010(n=72)$ and $2011(n=6)$ were evaluated as one time point.

${ }^{b}$ Breakpoints for susceptible and resistant isolates were according to the European Committee on Antimicrobial Susceptibility Testing (EUCAST; www.eucast.org/clinical_breakpoints).

'Breakpoints not stated by any organization. 


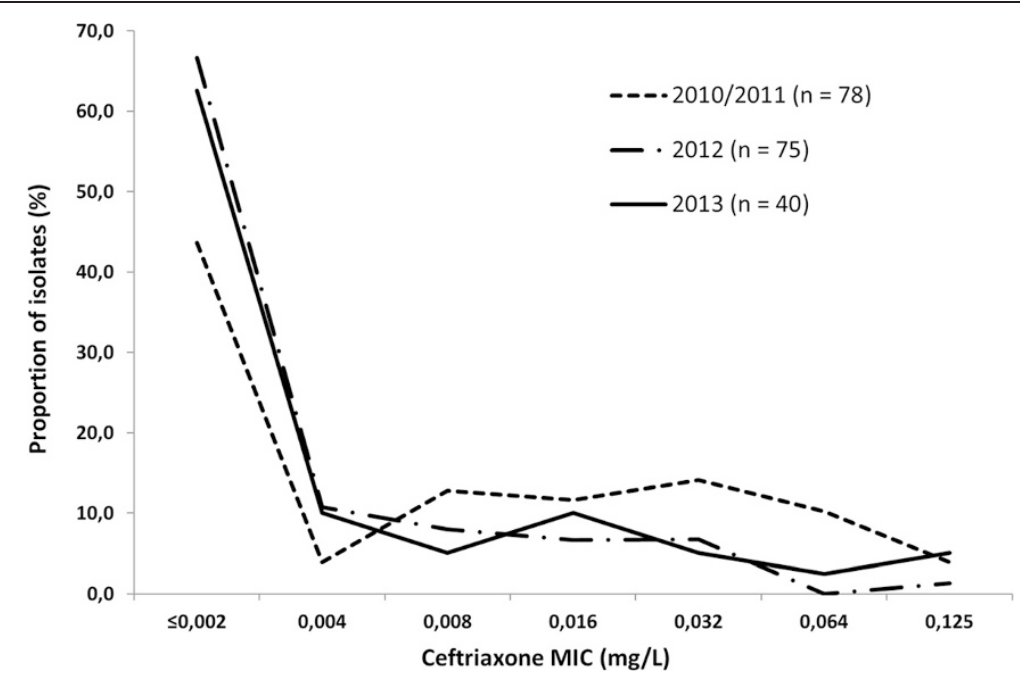

Figure 1 The distribution of minimum inhibitory concentrations (MICs) of ceftriaxone for Neisseria gonorrhoeae isolates ( $n=193$ ) cultured in Belarus from 2010 to 2013.

\section{Molecular epidemiological typing}

The 193 were assigned to 70 different NG-MAST STs. The most prevalent ST was ST1993 ( $\mathrm{n}=53,27.5 \%$ of isolates), followed by ST807 $(\mathrm{n}=13,6.7 \%)$, ST285 $(\mathrm{n}=8$, 4.1\%), ST9735 $(n=8,4.1 \%)$ and ST2873 $(n=6,3.1 \%)$. The remaining $\mathrm{STs}$ were represented by five (1 cluster), four $(n=3)$, three $(n=8)$, two $(n=11)$ and one $(n=42)$ isolates. Forty-three (61\%) of these STs were not previously described. In general, the most prevalent STs such as ST1993, ST807, ST285 and ST9735 had relatively low MICs of ceftriaxone. Notably, the cefixime resistant gonococcal isolate obtained in 2013 was assigned as ST3149.

\section{Discussion}

In the present study, the AMR in $N$. gonorrhoeae isolates cultured from 2010 to 2013, and molecular epidemiological characteristics (NG-MAST) of N. gonorrhoeae isolates in Belarus were examined. Previously, only one minor AMR study examining gonococcal isolates from Belarus in 2009 [28] has been internationally published. Accordingly, exceedingly limited phenotypic and genetic characteristics of the gonococcal strains spreading in Belarus have been previously published.

High prevalences of resistance were observed for previously recommended antimicrobials such as tetracycline (36\%), ciprofloxacin (28\%), and penicillin G (9\%). In accordance with the WHO recommendations regarding exclusion of an antimicrobial when the resistance level reaches $\geq 5 \%$ in the general population, none of these antimicrobials should be recommended for empirical firstline monotherapy of gonorrhoea in Belarus or in most other countries globally where resistance is prevalent [2,5-11]. In line with this, the results of the present surveillance initiated in 2009 [28] were also used to replace penicillin $\mathrm{G}$ with ceftriaxone as the first-line drug for empiric treatment of gonorrhoea in the national treatment guidelines in Belarus in late 2009 [29]. Interestingly, $\beta$-lactamase producing gonococcal strains appear to be exceedingly rare (1/193 isolates) in Belarus, which is also the case in some other independent countries of the former Soviet Union such as Russia [30-33]. In Belarus, where penicillin $G$ and other penicillin derivatives have been widely used for treatment of gonorrhoea at least until 2010, this may indicate that no imported $\beta$-lactamase producing gonococcal strains have been established and resulted in an endemic spread in Belarus. Resistance to azithromycin was also relatively prevalent (5\%), however, no isolates with high-level resistance to azithromycin ( $\mathrm{MIC} \geq 256 \mathrm{mg} / \mathrm{L}$ ), which have been described from several other countries [34-39], have yet been identified in Belarus. Furthermore, the resistance to azithromycin significantly declined from $12 \%$ in 2010 to $0 \%$ in 2013, which might reflect that azithromycin is rarely used for treatment of gonorrhoea and utilized in a controlled manner in the treatment of other STIs such chlamydial infection.

Since late 2009, in Belarus ceftriaxone $1 \mathrm{~g}$ single dose intramuscularly is the nationally recommended first-line treatment for uncomplicated anogenital and extragenital gonorrhoea [29]. In the present study, no ceftriaxone resistant isolates and only one $(0.5 \%)$ cefixime resistant isolate were identified. This is in contrast to the findings in the neighbouring country Russia, where according to the EUCAST breakpoints (www.eucast.org) $2.7 \%$ of isolates were resistant to ceftriaxone in 20092012 [33]. The reasons for the low MICs of ceftriaxone in Belarus are unknown. However, this might reflect that 
no gonococcal strain with decreased susceptibility or resistance to ESCs has been imported and/or managed to establish any domestic spread. Furthermore, in Belarus cefixime or other less potent oral ESCs have never been widely used for treatment of gonorrhoea. No resistance to spectinomycin was either found. This is also in contrast to the findings in Russia, where isolates with lowlevel resistance to spectinomycin are detected every year [31-33]. Worryingly, in Belarus despite that ceftriaxone $1 \mathrm{~g}$ single dose intramuscularly is the recommended first-line regimen penicillins, fluoroquinolones, macrolides, and other cephalosporins remain to be used in the routine gonorrhoea treatment. Furthermore, antimicrobials are easily available "over-the-counter", and this practice is crucial to abandon. The use of also less potent antimicrobials in the treatment of gonorrhoea, spread of gonococcal strains with decreased ESC susceptibility in Belarus and ESC resistance internationally, including in the neighbouring country Russia where also spectinomycin resistance are spreading, necessitate an enhanced surveillance of gonococcal AMR (with focus on ceftriaxone but also multidrug resistance), and ideally also treatment failures and molecular epidemiological characteristics in Belarus. There are also initiatives to improve the gonococcal AMR surveillance in Belarus, that is, to increase the number of isolates examined annually, the geographic representativeness by including additional regions, and the linkage to appropriate epidemiological data. In general, national and international support, including political and financial commitment, is essential to strengthen the gonococcal AMR surveillance in non-EU/EEA countries of the WHO European Region [2-4].

NG-MAST has been used in many countries worldwide; to monitor the national and international spread of gonococcal strains, including AMR clones, to identify transmission patterns within sexual networks, and to verify or falsify suspected treatment failures [26]. In Belarus, during 2010-2013 70 different NG-MAST STs among 193 isolates were identified. The substantial number of STs represented by only one isolate $(n=42)$ and STs that have not been previously described $(n=43 ; 61 \%)$ may be associated with the low number of cultured gonococcal isolates from each surveillance site, suboptimal diagnostics, lack of tracing of sexual contacts, and STs evolved locally in Belarus. Nevertheless, some major ST clusters of, e.g., ST1993 $(\mathrm{n}=53,27.5 \%$ of isolates), followed by ST807 $(\mathrm{n}=13$, 6.7\%), ST285 ( $\mathrm{n}=8,4.1 \%)$, and ST9735 $(\mathrm{n}=8,4.1 \%)$, were identified, which indicate some larger sexual transmission chains. No isolates assigned as ST1407, the internationally spread multidrug resistant gonococcal clone $[5,8,11,14,18,40-44]$, was identified from 2010 to 2013 in Belarus.

\section{Conclusions}

In Belarus, during 2010-2013 the heterogeneous gonococcal population showed high and relatively stable resistance levels to ciprofloxacin, tetracycline and penicillin G. The overall resistance to azithromycin was also relatively high, however, significantly declined from 2010 to 2013. One isolate was resistant to cefixime, but no resistance to ceftriaxone or spectinomycin was found. The results of the present surveillance initiated in 2009 [28] were also used to replace penicillin $G$ with ceftriaxone (1 g single dose intramuscularly) as the first-line drug for empiric treatment of gonorrhoea in the national treatment guidelines in Belarus in late 2009. Accordingly, based on the present study and resistance data from worldwide [2,5-11,19,30-33,40-42,45], ceftriaxone should be the only option for first-line empiric antimicrobial monotherapy of gonorrhoea in Belarus. Spectinomycin should be the alternative treatment option and only used when ceftriaxone is not available or the patient suffers from a severe $\beta$-lactam allergy. However, if pharyngeal gonorrhoea has not been excluded azithromycin is recommended to be added to the spectinomycin regimen. It is essential to further strengthen the surveillance of gonococcal AMR and ideally survey also treatment failures and molecular epidemiological genotypes in Belarus.

\section{Abbreviations \\ AMR: Antimicrobial resistance; ECDC: European centre for disease prevention and control; EEA: European economic area; ESC: Extended-spectrum cephalosporins; EU: European union; EUCAST: European committee on antimicrobial susceptibility testing; MIC: Minimum inhibitory concentration; NG-MAST: N. gonorrhoeae multiantigen sequence typing; ST: Sequence type; WHO: World Health Organization.}

Competing interests

The authors declare that they have no competing interests.

\section{Authors' contributions}

FL, LT, SG, AL, DG, IS, MD and MU designed and initiated the study. FL, SG, $D G, N P$, and NC coordinated and performed all the laboratory analyses. FL and $\mathrm{MU}$ analysed and interpreted all the data, and wrote a first draft of the paper. All authors read, commented on and approved the final manuscript.

\section{Acknowledgments}

This work was supported by the Örebro County Council Research Committee, the Foundation for Medical Research at Örebro University Hospital, the World Health Organization, the Eastern European Sexual and Reproductive Health (EE-SRH) Network, and the Swedish Institute (DBr00873/ 2011).

\section{Author details}

${ }^{1}$ The Republican Research and Practical Center for Epidemiology and Microbiology (RRPCEM), Minsk, Belarus. ${ }^{2} \mathrm{WHO}$ Collaborating Centre for Gonorrhoea and other Sexually Transmitted Infections, Swedish Reference Laboratory for Pathogenic Neisseria, Department of Laboratory Medicine, Microbiology, Örebro University Hospital, Örebro, Sweden. ${ }^{3}$ Mogilev Regional Dermato-Venerological Dispensary, Mogilev, Belarus. ${ }^{4}$ Minsk City Dermato-Venerological Dispensary, Minsk, Belarus. ${ }^{5}$ Vitebsk Regional Dermato-Venerological Dispensary, Vitebsk, Belarus. ${ }^{6}$ Department of Prevention and Control of Communicable Diseases, Uppsala County Council, Uppsala, Sweden. 
Received: 15 October 2014 Accepted: 15 January 2015

Published online: 31 January 2015

\section{References}

1. European Centre for Disease Prevention and Control. Sexually Transmitted Infections in Europe 2012. Stockholm: ECDC; 2014. Available at: http://www. ecdc.europa.eu/en/publications/Publications/sexually-transmitted-infectionseurope-surveillance-report-2012.pdf (Accessed: December 17, 2014).

2. Unemo M, Ison CA, Cole M, Spiteri G, van de Laar M, Khotenashvili L. Gonorrhoea and gonococcal antimicrobial resistance surveillance networks in the WHO European Region, including the independent countries of the former Soviet Union. Sex Transm Infect. 2013;89 Suppl 4:iv42-6.

3. Unemo M, Shipitsyna E, Domeika M. Recommended antimicrobial treatment of uncomplicated gonorrhoea in 2009 in 11 East European countries: implementation of a Neisseria gonorrhoeae antimicrobial susceptibility programme in this region is crucial. Sex Transm Infect. 2010;86:442-4.

4. Unemo M, Shipitsyna E, Domeika M. Gonorrhoea surveillance, laboratory diagnosis and antimicrobial susceptibility testing of Neisseria gonorrhoeae in 11 countries of the eastern part of the WHO European region. APMIS. 2011;119:643-9.

5. Unemo M, Shafer WM. Antimicrobial resistance in Neisseria gonorrhoeae in the 21st Century: past, evolution, and future. Clin Microbiol Rev. 2014;27:587-613.

6. Tapsall JW, Ndowa F, Lewis DA, Unemo M. Meeting the public health challenge of multidrug- and extensively drug-resistant Neisseria gonorrhoeae. Expert Rev Anti Infect Ther. 2009;7:821-34.

7. Lewis DA. The gonococcus fights back: is this time a knock out? Sex Transm Infect. 2010;86:415-21.

8. Unemo M, Nicholas RA. Emergence of multi-drug resistant, extensively drug-resistant and untreatable gonorrhea. Future Microbiol. 2012;7:1401-22.

9. Whiley DM, Goire N, Lahra MM, Donovan B, Limnios AE, Nissen MD, et al. The ticking time bomb: escalating antibiotic resistance in Neisseria gonorrhoeae is a public health disaster in waiting. J Antimicrob Chemother 2012;67:2059-61.

10. Bolan GA, Sparling PF, Wasserheit JN. The emerging threat of untreatable gonococcal infection. N Engl J Med. 2012;366:485-7.

11. Spiteri G, Cole M, Unemo M, Hoffmann S, Ison C, van de Laar M. The European Gonococcal Antimicrobial Surveillance Programme (Euro-GASP)-a sentinel approach in the European Union (EU)/European Economic Area (EEA). Sex Transm Infect. 2013;89 Suppl 4:iv16-8.

12. Tapsall J, Read P, Carmody C, Bourne C, Ray S, Limnios A, et al. Two cases of failed ceftriaxone treatment in pharyngeal gonorrhoea verified by molecular microbiological methods. J Med Microbiol. 2009;58:683-7.

13. Unemo M, Golparian D, Hestner A. Ceftriaxone treatment failure of pharyngeal gonorrhoea verified by international recommendations, Sweden, July 2010. Euro Surveill. 2011;16(6):19792.

14. Unemo M, Golparian D, Potocnik M, Jeverica S. Treatment failure of pharyngeal gonorrhoea with internationally recommended first-line ceftriaxone verified in Slovenia, September 2011. Euro Surveill. 2012;17(25):20200.

15. Ohnishi M, Golparian D, Shimuta K, Saika T, Hoshina S, Iwasaku K, et al. Is Neisseria gonorrhoeae initiating a future era of untreatable gonorrhea?: detailed characterization of the first strain with high-level resistance to ceftriaxone. Antimicrob Agents Chemother. 2011;55:3538-45.

16. Chen YM, Stevens K, Tideman R, Zaia A, Tomita T, Fairley CK, et al. Failure of ceftriaxone $500 \mathrm{mg}$ to eradicate pharyngeal gonorrhoea, Australia. J Antimicrob Chemother. 2013;68:1445-7.

17. Read PJ, Limnios EA, McNulty A, Whiley D, Lahra LM. One confirmed and one suspected case of pharyngeal gonorrhoea treatment failure following 500 mg ceftriaxone in Sydney, Australia. Sex Health. 2013;10:460-2.

18. Golparian D, Ohlsson A, Janson H, Lidbrink P, Richtner T, Ekelund O, et al. Four treatment failures of pharyngeal gonorrhoea with ceftriaxone $(500 \mathrm{mg})$ or cefotaxime (500 mg), Sweden, 2013 and 2014. Euro Surveill. 2014;19(30):pii:20862.

19. World Health Organization (WHO), Department of Reproductive Health and Research. Global action plan to control the spread and impact of antimicrobial resistance in Neisseria gonorrhoeae. Geneva: WHO; 2012. p. 1-36. Available at: http://www.who.int/reproductivehealth/publications/rtis/9789241503501 (Accessed: December 17, 2014).

20. Ndowa F, Lusti-Narasimhan M, Unemo M. The serious threat of multidrug-resistant and untreatable gonorrhoea: the pressing need for global action to control the spread of antimicrobial resistance, and mitigate the impact on sexual and reproductive health. Sex Transm Infect. 2012;88:317-8.

21. European Centre for Disease Prevention and Control (ECDC). Response plan to control and manage the threat of multidrug-resistant gonorrhoea in Europe. Stockholm: ECDC; 2012. p. 1-23. Available at: http://www.ecdc.europa.eu/en/ publications/Publications/1206-ECDC-MDR-gonorrhoea-response-plan.pdf (Accessed: December 17, 2014).

22. Domeika M, Savicheva A, Sokolovskiy E, Ballard R, Unemo M, Eastern European Network for Sexual and Reproductive Health (EE SRH Network). Quality enhancements and quality assurance of laboratory diagnosis of sexually transmitted infections in Eastern Europe. Int J STD AIDS. 2009:20:365-7.

23. Unemo M, Fasth O, Fredlund H, Limnios A, Tapsall JW. Phenotypic and genetic characterization of the 2008 WHO Neisseria gonorrhoeae reference strain panel intended for global quality assurance and quality control of gonococcal antimicrobial resistance surveillance for public health purposes. J Antimicrob Chemother. 2009;63:1142-51.

24. Tapsall JW, Cheng JK. Rapid identification of pathogenic species of Neisseria by carbohydrate degradation tests: importance of glucose in media used for preparation of inocula. Br J Ven Dis. 1981;14:249-52.

25. Martin IM, Ison CA, Aanensen DM, Fenton KA, Spratt BG. Rapid sequence-based identification of gonococcal transmission clusters in a large metropolitan area. J Infect Dis. 2004;189:1497-505.

26. Unemo M, Dillon JA. Review and international recommendation of methods for typing Neisseria gonorrhoeae isolates and their implications for improved knowledge of gonococcal epidemiology, treatment, and biology. Clin Microbiol Rev. 2011;24:447-58.

27. Unemo M, Sjöstrand A, Akhras M, Gharizadeh B, Lindbäck E, Pourmand N, et al. Molecular characterization of Neisseria gonorrhoeae identifies transmission and resistance of one ciprofloxacin-resistant strain. APMIS. 2007;115:231-41.

28. Glazkova S, Golparian D, Titov L, Pankratova N, Suhabokava N, Shimanskaya I, et al. Antimicrobial susceptibility/resistance and molecular epidemiological characteristics of Neisseria gonorrhoeae in 2009 in Belarus. APMIS. 2011;119:537-42

29. Ministry of Health in Belarus: Annex 2 to the Order of Health Ministry of October 29, 2009 № 1020 "Clinical diagnosis and treatment protocol of patients with sexually transmitted infections". [In Russian]

30. Unemo M, Vorobieva V, Firsova N, Ababkova T, Leniv I, Haldorsen BC, et al. The Neisseria gonorrhoeae population transmitted in Arkhangelsk, Russia in 2004 - phenotypic and genotypic heterogeneity. Clin Microbiol Infect. 2007;13:873-8.

31. Kubanova A, Kubanov A, Frigo N, Sidorenko S, Priputnevich T, Vachnina T, et al. National surveillance of antimicrobial susceptibility in Neisseria gonorrhoeae in 2005-2006 and recommendations of first-line antimicrobial drugs for gonorrhoea treatment in Russia. Sex Transm Infect. 2008;84:285-9.

32. Kubanova A, Frigo N, Kubanov A, Sidorenko S, Lesnaya I, Polevshikova S, et al. The Russian gonococcal antimicrobial susceptibility programme (RU-GASP) - national resistance prevalence in 2007 and 2008, and trends during 2005-2008. Euro Surveill. 2010;15(14):19533.

33. Kubanova A, Kubanov A, Frigo N, Solomka V, Semina V, Vorobyev D, et al. Russian gonococcal antimicrobial susceptibility programme (RU-GASP) resistance in Neisseria gonorrhoeae during 2009-2012 and NG-MAST genotypes in 2011 and 2012. BMC Infect Dis. 2014;14(1):342.

34. Palmer HM, Young $H$, Winter A, Dave J. Emergence and spread of azithromycin-resistant Neisseria gonorrhoeae in Scotland. J Antimicrob Chemother. 2008:62:490-4.

35. Chisholm SA, Dave J, Ison CA. High-level azithromycin resistance occurs in Neisseria gonorrhoeae as a result of a single point mutation in the 23S rRNA genes. Antimicrob Agents Chemother. 2012;54:3812-6.

36. Starnino S, Stefanelli P, Neisseria gonorrhoeae Italian Study Group I. Azithromycin-resistant Neisseria gonorrhoeae strains recently isolated in Italy. J Antimicrob Chemother. 2009;63:1200-4.

37. Galarza PG, Abad R, Canigia LF, Buscemi L, Pagano I, Oviedo C, et al. New mutation in $23 \mathrm{~S}$ rRNA gene associated with high level of azithromycin resistance in Neisseria gonorrhoeae. Antimicrob Agents Chemother. 2010;54:1652-3.

38. Katz AR, Komeya AY, Soge OO, Kiaha MI, Lee MV, Wasserman GM, et al. Neisseria gonorrhoeae with high-level resistance to azithromycin: case report of the first isolate identified in the United States. Clin Infect Dis. 2012:54:841-3. 
39. Unemo M, Golparian D, Hellmark B. First three Neisseria gonorrhoeae isolates with high-level resistance to azithromycin in Sweden: a threat to currently available dual-antimicrobial regimens for treatment of gonorrhea? Antimicrob Agents Chemother. 2013;58:624-5.

40. Jeverica S, Golparian D, Maticic M, Potočnik M, Mlakar B, Unemo M. Phenotypic and molecular characterization of Neisseria gonorrhoeae isolates from Slovenia, 2006-12: rise and fall of the multidrug-resistant NG-MAST genogroup 1407 clone? J Antimicrob Chemother. 2014;69:1517-25.

41. Mlynarczyk-Bonikowska B, Serwin AB, Golparian D, Walter de Walthoffen S, Majewski S, Koper M, et al. Antimicrobial susceptibility/resistance and genetic characteristics of Neisseria gonorrhoeae isolates from Poland, 2010-2012. BMC Infect Dis. 2014;14(1):65.

42. Ison CA, Town K, Obi C, Chisholm S, Hughes G, Livermore DM, et al. Decreased susceptibility to cephalosporins among gonococci: data from the Gonococcal Resistance to Antimicrobials Surveillance Programme (GRASP) in England and Wales, 2007-2011. Lancet Infect Dis. 2013;13:762-8.

43. Chisholm SA, Unemo M, Quaye N, Johansson E, Cole MJ, Ison CA, et al. Molecular epidemiological typing within the European gonococcal antimicrobial resistance surveillance programme reveals predominance of a multidrug-resistant clone. Euro Surveill. 2013;18(3):20358.

44. Grad YH, Kirkcaldy RD, Trees D, Dordel J, Harris SR, Goldstein E, et al. Genomic epidemiology of Neisseria gonorrhoeae with reduced susceptibility to cefixime in the USA: a retrospective observational study. Lancet Infect Dis. 2014;14:220-6.

45. Bala M, Kakran M, Singh V, Sood S, Ramesh V. Monitoring antimicrobia resistance in Neisseria gonorrhoeae in selected countries of the WHO South-East Asia Region between 2009 and 2012: a retrospective analysis. Sex Transm Infect. 2013;89 Suppl 4:iv28-35.

\section{Submit your next manuscript to BioMed Central and take full advantage of:}

- Convenient online submission

- Thorough peer review

- No space constraints or color figure charges

- Immediate publication on acceptance

- Inclusion in PubMed, CAS, Scopus and Google Scholar

- Research which is freely available for redistribution 